\title{
Discussion of the Causes, Effect and Potential Methods of Alleviating Patient Anxiety When Undergoing Magnetic Resonance Imaging (MRI)
}

\author{
Zyad Mansour Almutlaq \\ King Saud bin Abdulaziz University for Health Sciences, College of Applied Medical Sciences, Radiology \\ Department \\ Corresponding Author: Zyad Mansour Mohammed Almutlaq, Email: zy.almutlaq@ gmail.com, Telephone number: +966550280067.
}

\begin{abstract}
Background: In performing an MRI examination the patient may feel anxious due to the nature of the MRI scan as the patient would be inserted into the narrow bore of the MRI scanner and would be asked to be immobile during the examination period for approximately 20 to 90 minutes depending on the scan type. Patient feeling anxious may also be evoked by several causes and consequently may develop into anxiety reactions by the patient either before, during, or after the scan. Aim: This paper aim to study the issues surrounding the anxiety in patients undergoing MRI examinations by identifying the prominent causes, outlining the possible effects of anxiety, discussing the main potential methods for alleviating patient's anxiety. Materials and methods: This review is conducted by using a comprehensive search of PubMed, Google Scholar, and Elsevier, August 1993, through March 2018. The following search terms were used: causes of anxiety in MRI, claustrophobia and MRI, effects of anxiety in MRI, alleviation of anxiety in MRI patient. Conclusion: Patient's anxiety can pose problems that may impede the process of the MRI examination. Patient's anxiety may have adverse effects on both patient and the healthcare institution as it can cause financial losses for the healthcare institutions. However, sedation techniques, acoustic noise reduction, and adequate patient preparation for the MRI scan are possible methods that can alleviate patient's anxiety.
\end{abstract}

Key words: MRI, Medical Imaging, Patient Anxiety, Claustrophobia.

\section{INTRODUCTION}

Magnetic resonance imaging (MRI) is one of the main medical imaging modalities along with Computed Tomography (CT), Ultrasound (US), XRays, Single-Photon Emission Computed Tomography (SPECT), and Interventional radiography (IVR). The prominence of MRI has increased since 1980 until it has become a common diagnostic procedure these days, where MRI is an advanced imaging technique capable of imaging the human body internal organs and detecting various abnormalities, with highresolution details ${ }^{(1-2)}$. MRI is distinguished from other medical imaging modalities using a high magnetic field with radio waves for obtaining a diagnostic image instead of exposing a patient to an ionizing radiation ${ }^{(3)}$. Therefore, it is considered biologically safe. Moreover, MRI is the best imaging tool that has the ability to provide a clear contrast between the soft tissues in the image ${ }^{(4)}$. Thus, it helps diagnosing in an accurate way various types of disorders within body organs that not functioning in a normal orderly healthy manner.

In performing an MRI scan, the patient will be inserted into the bore of the MRI machine. Subsequently, the patient will be asked to be immobile during the examination for approximately 20 to 90 minutes depending on the scan type. However, due to the nature of the MRI scan, the patient may feel anxious. This feeling which is evoked by several causes may develop into anxiety reactions by the patient either before, during, or after the examination.

\section{AIM AND METHOD}

Taking into account the above-mentioned points, this paper aimed at discussing the issues of anxiety in patients undergoing MRI examinations by identifying the prominent causes of anxiety and then outlining the possible effects of anxiety. This will be followed by a brief discussion of the main potential methods for alleviating patient's anxiety.

\section{Causes of patients' anxiety: \\ Claustrophobia}

Claustrophobia, a severe form of anxiety, is one of the most common causes of apprehension amongst patients undergoing MRI examinations. McIsaac et al. ${ }^{(1)}$ recognised that approximately $37 \%$ of patients who are booked for MRI scans have different levels of claustrophobia. Also, Dewey et al.

(5) declared that from $1 \%$ to $15 \%$ of MRI participants express claustrophobia. Although there is a distinct variation between the proportions mentioned in the above sources, these percentages indeed represent a large number if they are converted to real numbers, particularly as there are thousands of patients during the year who are referred to the medical imaging to be scanned by MRI ${ }^{(2)}$. 
In terms of the influential factors that could motivate the incidence of claustrophobia, Eshed et $\boldsymbol{a l}{ }^{(2)}$ argued that fright of being in a finite area or an enclosed space can induce the claustrophobia. McIsaac et al. ${ }^{(1)}$ illustrated that claustrophobia is not a fear of enclosed space itself as much as a fear of what may occur in that place. Therefore, the claustrophobia can be defined as a pessimistic expectation of outcomes of being in a confined area. Moreover, it comprises two components: a panic of restricted motion and fearfulness of suffocation, and both exist in an MRI examination.

The first component (immobilisation) can be apparent in each MRI scan as a patient must remain motionless inside the machine during the examination, and the second element is evoked by a feeling of confinement inside the narrow bore of the MRI scanner which reduces the air flow to the patient ${ }^{(1)}$. In a study conducted in 1997 among patients who underwent MRI scans, 38 patients out of 137 acknowledged that the cramped space of the MRI bore is the most unpleasant factor that evoked their fears ${ }^{(6)}$. Other authors in addition to the above elements, mentioned that claustrophobia may involve fear that the scanner could cause harm, and fear of the loss of self-control ${ }^{(7)}$.

\section{Patient position}

The method of patient insertion into an MRI machine has a close correlation with the incidence of claustrophobia and a strong impact on the level of a patient's anxiety. Usually, the patient's head is inserted first into the bore of the MRI machine, when the scan is required to examine the patient's head or upper part of the body and this manner is called head-first whereas if the part to be examined is one of the lower extremities, the patient's feet will be inserted first into the scanner, and this method is termed feet-first ${ }^{(1)}$. A substantial body of research indicated that claustrophobic reactions are highly associated with most of the patients who are inserted into the scanner with the head-first method, and their probability of experiencing growing anxiety during the examination is high ${ }^{(1,25)}$.

In an eight-year analytical study, Dewey et al. (5) found that out of 45,998 patients who had been introduced into the MRI machine with the head-first manner, 986 of them had suffered from claustrophobia. Moreover, Eshed et al. ${ }^{(2)}$ concluded that 34 out of 1968 participants who underwent MRI examinations for Head and Neck had experienced claustrophobia. This is consistent with the previous study and provides a probable logical interpretation of the reason for patient's anxiety and the confinement feeling inside the scanner. Contrary to this, the sense of confinement was lower in patients who had undergone an MRI scan for their legs, which explicates the decreased incidence of claustrophobic reactions in an MRI exam when the lower part of the body is scanned. However, Thorpe et $\boldsymbol{a l}{ }^{(7)}$ claimed that there is no correlation between the method of positioning the patient into the scanner and the level of a patient's anxiety.

\section{Acoustic noise}

The acoustic noise which results from the MRI machine is a significant factor affecting the patient's feeling. During MRI examinations, the scanner produces various noisy sounds, which are similar to a series of raucous knocking, ringing, or loud chirping. These sounds often have a negative impact on the patient's experience during the scan which may cause anxiety to the patient. Three surveys evaluated the impact of the acoustic noise from the scanner on the patient's experience of the MRI scan. The results showed that 73 out of 257 patients indicated that sounds were unacceptable. Moreover, $11.5 \%$ of subjects stated that the noise levels were very loud ${ }^{(6,8,9)}$.

\section{Inadequate preparation}

Insufficient preparation of the patient for an MRI examination can increase the chances of incidence of anxiety reactions during the scan. Often, the patient is surprised by the scanner's shape and the narrowed space of its bore, as surprised by the restricted movement allowed during the examination and loud noisy sounds issued by the MRI machine. These surprises could be resulting from the less acceptability of the imaging procedure with consequent generation of anxiety with the patient. In a qualitative study of the causes of patients' anxiety during MRI scans, $42 \%$ of patients reported that lack of detailed information about the examination and its nature was the main cause of their disquiet ${ }^{(10)}$.

\section{The effects of patients' anxiety:}

\section{Alter the image quality}

Patient's anxiety has substantial effects. In terms of its influence on examination outcomes, patient's anxiety can alter the image quality negatively. During MRI procedure, the patient must remain motionless in order to obtain a high-quality diagnostic image. The patient's movement, albeit slight motion, will degrade 
acquired image quality and consequently the radiologist may not be able to interpret it accurately. A study revealed an association between physiological responses and anxiety, where the physiological responses to the patient's body muscles are affected by the level of anxiety. Moreover, an increased level of anxiety would increase body's movement and thus reduce the image quality. Also, the study found that 38 out of the 279 anxious patients who underwent MRI scans have moved during their examinations, and these movements have constrained the diagnostic abilities in 19 of them ${ }^{(6)}$. However, another study showed that 8 out of 33 patients have moved during examinations due to their anxiety without affecting the quality of the diagnosis ${ }^{(11)}$. In fact Klaming et al. ${ }^{(12)}$ believed that anxiety does not contribute to the patient's movement, but on the contrary, it helps to keep the patient motionless throughout the examination.

\section{Early scan termination and induce claustrophobia}

Severe anxiety reactions can impede the patient from staying still during the examination and lead to an early termination of the scan. Restless patients frequently tend to terminate their scan before it is completed, due to their inability to continue the examination ${ }^{(13)}$. According to some studies, early termination rates of the scan range from $1.22 \%$ to $39 \%$ in anxious patients ${ }^{(2,13)}$. Consequently, incomplete examinations have serious effects on both the patients and the healthcare institutions.

An incomplete scan due to anxiety may result in psychological effects on the patient. The fear of enclosed spaces may develop in the patient after withdrawal from an MRI scan if the patient shows severe anxiety reactions during the examination. A qualitative study found that approximately $10 \%$ of patients who did not complete their scans become more anxious in confined spaces than in the former ${ }^{(14)}$. Additionally, McIsaac et al. ${ }^{(1)}$ revealed that 24 out of 80 claustrophobic patients indicated that their fears of the confined spaces have increased after withdrawing from the examination. However, minimal studies claimed that withdrawing from the MRI scan by a non-claustrophobic patient due to the discomfort of the narrow space could induce claustrophobia in that patient ${ }^{(1,7)}$. According to Sarji et al. (15), 50\% of patients who had withdrawn from their scan had never felt claustrophobia before their first experience of an MRI examination.

\section{Patient no-shows}

Patient's anxiety may contribute to the absence from the examination, thus depriving the patient of the opportunity to benefit from the diagnosis of illness. A study to evaluate the number of patients who did not attend for their appointments in one of the university hospitals revealed that the average absence of the majority of the patients was 4 appointments. The study also showed that the reasons for the absence of these patients varied between concerns of the unknown or apprehension of the diagnostic result, fear of discomfort during the examination, and anticipatory anxiety ${ }^{(13)}$. In another extended study conducted in three hospitals, Norbash et al. ${ }^{(13)}$ found that the proportion of patients who failed to attend their MRI appointments in one year was $11 \%$.

\section{Financial losses}

The absence of the patient from the examination or withdrawal from it causes considerable financial losses on health institutions, where the cost of the MRI scans worldwide ranges from $\$ 250$ to $\$ 500$ ${ }^{(5,13,17)}$. In a study carried out by Dewey et al. ${ }^{(5)}$, showed that the financial losses in a hospital amounted to 84,250 dollars, because of the absence of 337 patients for their examinations. On a wider scale, Norbash et $\boldsymbol{a l} .^{(13)}$ reported that the annual financial waste in all US health institutions is estimated at 310 million dollars as a result of 700,000 incomplete MRI scans. However, the financial losses can be reduced by addressing the origin of the problem, which is the patient's anxiety through the application of one of the three following methods to alleviate it.

\section{Potential methods to alleviate patients' anxiety: \\ Patient sedation}

Usually, patients with severe anxiety reactions are sedated in most hospitals, where it provides a solution for patients who need to be scanned by MRI despite their fears or anxiety. The sedation can make the patient in a state of calm and conscious, where the sedated patient remains able to breathe without interference and able to respond to verbal orders received. Therefore, patient sedation is considered as a technique in which health institutions can reduce the amount of financial loss. Moreover, the patient could benefit from the diagnostic accuracy of high-quality MRI images ${ }^{(16-20)}$. In a study on the effectiveness of sedation techniques in patients with a history of anxiety reactions during previous MRI scan, 28 out of 30 sedated patients completed their examination 
successfully ${ }^{(20)}$. In another study conducted by Hollenhorst et al. ${ }^{(18)}$, it was found that sedation helped 27 claustrophobic patients to remain motionless and they completed the MRI scans successfully by reducing their fear levels.

Patient sedation has many drawbacks involving potential risks, additional expenses, long waiting times. Potential risks include the adverse effects that may be induced by sedation to a patient's heart and respiratory system. The additional expenses involve the need for nurses' anaesthetist and an anaesthesiologist, an MRIcompatible anaesthesia machine, and medications.

The waiting time for an MRI scan of the patient who requires sedation is very long, because of many requests for sedation concurrent with the shortage of anaesthesia staff, and limited available booking times, which may deteriorate the patient's health condition ${ }^{(19)}$.

\section{Acoustic noise reduction}

Reducing noise levels can significantly improve patient's comfort during the MRI examination. The manufacturers of MRI machines have adjusted the noise levels produced by the scanners that may contribute to patient's anxiety, where the noise levels in new technologies have been reduced by $97 \%$ (5) Although new MRI machines still produce noise, this adjustment has had a profound impact on ameliorating the patient's experience. A questionnaire study on the influence of the acoustic noise reduction on the patient's experience during an MRI scan showed that noise levels were acceptable for 12 out of 21 participants ${ }^{(9)}$. Minimizing the noise levels can alleviate patient's anxiety and thus reduce the possibility of anxiety reactions during the MRI examination. Dewey et al. ${ }^{(5)}$ inferred that acoustic noise reduction can reduce the incidence of patients' anxiety reactions by threefold.

\section{Sufficient preparation}

This method involves providing the patient with basic information describing the shape of the MRI machine, noise levels, and temperature of the examination room. This can be accomplished by an instructional brochure that afforded to the patient prior to the examination day. Adequate preparation also includes providing the patient with detailed information on the nature of the examination, its usefulness and importance, its duration, and instructions to be followed during the scan ${ }^{(10)}$.

Preparing the patient adequately for an MRI examination has a significant impact on the patient's experience, particularly that who has anticipatory anxiety, where the level of anxiety decreases when affording the patient sufficient information about the scan ${ }^{(3,21)}$. A study aimed at measuring the effect of proper patient preparation on anxious patients found that 74 out of 75 patients completed their examinations successfully due to receiving a detailed explanation of the scan prior to the experience. Therefore, it seems clear that adequate patient preparation has a significant influence on reducing patient's anxiety ${ }^{(10)}$.

\section{CONCLUSION}

It is possible to conclude that patient anxiety can pose problems that may impede the process of the MRI examination. This paper has indicated that the fear of the narrow space of the bore of the MRI machine, the various noisy sounds of the scanner, and inadequate preparation of the patient before the examination are the main prominent factors contributing to anxiety generation in the patient. This paper has explained that severe patient's anxiety may have adverse effects on both patient and the healthcare institution. Anxiety may cause the patient to move during the examination, which may affect the acquired image quality and the diagnostic accuracy. Also, the patient may withdraw or be absent from the examination for the reasons of anxiety. Thus, patients' anxiety can cause financial losses for the healthcare institutions due to the high financial cost of the MRI scans. Additionally, this paper has discussed the effectiveness of the sedation techniques, acoustic noise reduction, and adequate patient preparation for the MRI scan as potential methods that can alleviate patient's anxiety. Due to words count constraints, this paper cannot provide a comprehensive review of the causes of the patients' anxiety and the possible solutions to alleviate this issue. Moreover, as a result of a lack in the literatures related to this subject, further studies with special consideration and attention to the main causes of anxiety and possible reduction/alleviation are greatly required.

\section{REFERENCES}

1. McIsaac H, Thordarson D, Shafran R,
Rachman S and Poole G (1998):
Claustrophobia and the magnetic resonance
imaging procedure. Journal of Behavioral
Medicine, 21(3): $255-268$.


2. Eshed I, Althoff C, Hamm B and Hermann K (2007): Claustrophobia and premature termination of magnetic resonance imaging examinations. Journal of Magnetic Resonance Imaging, 26(2): 401-404.

3. Tazegul G, Etcioglu E, Yildiz F, Yildiz R and Tuney D (2015): Can MRI related patient anxiety be prevented? Journal of Magnetic Resonance Imaging, 33(1): 180-183.

4. United States Food and Drug Administration (2018): Medical Imaging. [Online]. [Accessed 23 May 2018]. Available from: https:// www. fda.gov/Radiation-Emitting Products/ Radiation Emitting Products and Procedures/ Medical Imaging/ MRI/default.htm.

5. Dewey M, Schink T, and Dewey C (2007): Claustrophobia during magnetic resonance imaging: Cohort study in over 55,000 patients. Journal of Magnetic Resonance Imaging, 26(5): 1322-1327.

6. Dantendorfer K, Amering M, Bankier A, Helbich T, Prayer D, Youssefzadeh S, Alexandrowicz R, Imhof H, and Katschnig H (1997): A study of the effects of patient anxiety, perceptions and equipment on motion artifacts in magnetic resonance imaging. Magnetic Resonance Imaging, 15(3): 301-306.

7. Thorpe $S$, Salkovskis $P$, and Dittner A (2008): Claustrophobia in MRI: the role of cognitions. Magnetic Resonance Imaging, 26(8): 1081-1088.

8. Harris L, Cumming S, and Menzies R (2004): Predicting anxiety in magnetic resonance imaging scans. International Journal of Behavioral Medicine, 11(1): 1-7.

9. McNulty J, and McNulty S (2009): Acoustic noise in magnetic resonance imaging: An ongoing issue. Radiography, 15(4): 320-326.

10. Meléndez J (1993): Anxiety-Related Reactions Associated With Magnetic Resonance Imaging Examinations. The Journal of the American Medical Association, 270(6): 745-747.

11. Bangard C, Paszek J, Berg F, Eyl G, Kessler, J, Lackner K, and Gossmann A (2007): MR imaging of claustrophobic patients in an open 1.0T scanner: Motion artifacts and patient acceptability compared with closed bore magnets. European Journal of Radiology, 64(1): 152-157.
12. Klaming L, Minde $D$, Weda $H$, Nielsen $T$, and Duijm L (2015): The relation between anticipatory anxiety and movement during an MR examination. Academic Radiology, 22(12): 1571-1578.

13. Norbash A, Yucel K, Yuh W, Doros G, Ajam A, Lang E, Pauker S, and Mayr N (2016): Effect of team training on improving MRI study completion rates and no-show rates. Journal of Magnetic Resonance Imaging, 44(4): 1040-1047.

14. Lukins R, Davan I, and Drummond P (1997): A cognitive behavioural approach to preventing anxiety during magnetic resonance imaging. Journal of Behavior Therapy and Experimental Psychiatry, 28(2): 97-104.

15. Sarji S, Abdullah B, Kumar G, Tan A, and Narayanan $P$ (1998): Failed magnetic resonance imaging examinations due to claustrophobia. Australasian Radiology, 42(4): 293-295.

16. Murphy K, and Brunberg J (1997): Adult claustrophobia, anxiety and sedation in MRI. Magnetic Resonance Imaging, 15(1): 51-54.

17. Phillips S, and Deary I (1995): Interventions to alleviate patient anxiety during magnetic resonance imaging: A review. Radiography, 1(1): 29-34.

18. Hollenhorst J, Münte S, Friedrich L, Heine J, Leuwer $M$, Becker $H$, and Piepenbrock $S$ (2001): Using Intranasal Midazolam Spray to Prevent Claustrophobia Induced by MR Imaging. American Journal of Roentgenology, 176(4): 865-868.

19. Lemaire C, Moran G, and Swan H (2009): Impact of audio/visual systems on pediatric sedation in magnetic resonance imaging. Journal of Magnetic Resonance Imaging, 30(3): 649-655.

20. Loh $P$, Ariffin M, Rai V, Lai L, Chan L, and Ramli N (2016): Comparing the efficacy and safety between propofol and dexmedetomidine for sedation in claustrophobic adults undergoing magnetic resonance imaging (PADAM trial). Journal of Clinical Anesthesia, 34(1): 216-222.

21. Tornqvist E, Mansson A, Larsson E, and Hallstrom I (2006): It's like being in another world - patients' lived experience of magnetic resonance imaging. Journal of Clinical Nursing, 15(8): 954-961. 